\title{
Refractory Epithelioid Sarcoma
}

National Cancer Institute

\section{Source}

National Cancer Institute. Refractory Epithelioid Sarcoma. NCI Thesaurus. Code C162722.

An epithelioid sarcoma that does not respond to treatment. 